\title{
Separable Determination of the Fixed Point Property of Convex Sets in Banach Spaces
}

\author{
Qingxia $\mathrm{Li}^{1, *}$, Lili Su${ }^{2}$ and Qian $\mathrm{Wei}^{2}$ \\ ${ }^{1}$ Quanzhou Preschool Education College, Quanzhou 362000, Fujian, P.R. China \\ 2 School of Mathematical Sciences, Xiamen University, Xiamen 361005, Fujian, \\ P.R. China.
}

Received 15 July 2015; Accepted 15 September 2015

\begin{abstract}
In this paper, we first show that for every mapping $f$ from a metric space $\Omega$ to itself which is continuous off a countable subset of $\Omega$, there exists a nonempty closed separable subspace $S \subset \Omega$ so that $\left.f\right|_{S}$ is again a self mapping on $S$. Therefore, both the fixed point property and the weak fixed point property of a nonempty closed convex set in a Banach space are separably determined. We then prove that every separable subspace of $c_{0}(\Gamma)$ (for any set $\Gamma$ ) is again lying in $c_{0}$. Making use of these results, we finally presents a simple proof of the famous result: Every non-expansive self-mapping defined on a nonempty weakly compact convex set of $c_{0}(\Gamma)$ has a fixed point.
\end{abstract}

AMS subject classifications: 52B10, 65D18, 68U05, 68U07

Key words: Non-expansive mapping, weakly compact convex set, fixed point, Banach space.

\section{Introduction}

A mapping $f$ from a nonempty set $D$ to itself is said to be a self-mapping of $D$. If $D=$ $(D, d)$ is a metric space, then we call the mapping $f$ "Lipschitz" whenever there exists a constant $L>0$, so that

$$
d(f(x), f(y)) \leq L d(x, y), \quad \forall x, y \in D .
$$

In this case, we also say precisely that $f$ is $L$-Lipschitz on $D$. A mapping $f: D \rightarrow D$ is nonexpansive if it is 1-Lipschitz.

We say that a nonempty closed convex set $C$ of a Banach space $X$ has the fixed point property, if every nonexpansive mapping $f$ defined on each nonempty closed bounded convex subset $D$ of $C$ has a fixed point, i.e. there is $x_{0} \in D$ so that $f\left(x_{0}\right)=x_{0}$. Mathematicians also often consider the weak fixed point property of a closed convex set $C$ of

${ }^{*}$ Corresponding author. Email address: $1220950678 @ q q . c o m$ (L. Su), 1173806177@qq.com (Q. Wei) 
a Banach space $X$ : a Banach space $X$ has the weak fixed point property, if every nonexpansive self-mapping defined on a nonempty weakly compact convex set of $X$ has a fixed point. As is well known, the metric fixed point theory is a well-developed branch of fixed point theory, which not only has its own methods and problems, but also relates with other fields such as geometry of Banach spaces, integral and differential equations, multivalued analysis and so on. See, for example, Kirk [1].

Denote $c_{0}=\left\{\left(x_{n}\right)_{n \in \mathbb{N}}: \lim _{n \rightarrow \infty} x_{n}=0, \quad\|x\|_{\infty}=\sup _{n}\left|x_{n}\right|\right\}$. Let $\Gamma$ be a nonempty set and $c_{0}(\Gamma)$ denote the Banach space(supremum norm) of all real-valued function $x$ on $\Gamma$, such that for any $\varepsilon>0,\{r \in \Gamma,|x(r)|>\varepsilon\}$ is finite, or, equivalently, $c_{0}(\Gamma)$ consists of all those functions defined on the set $\Gamma$ with countable support and with its range as a null sequence. The spaces $c_{0}(\Gamma)$ have received renewed interest, because of a powerful mapping theorem of Lindenstrauss [2]: (1) They are weakly compactly generated Banach spaces; and (2) if $E$ is a weakly compactly generated Banach space, then there exists a set $\Gamma$ and a continuous one-to-one linear map $T$ of $E$ into $c_{0}(\Gamma)$.

Maurey $([3], 1980)$ first showed that $c_{0}$ has weak fixed point property by means of ultrapower techniques. Later, Odell and Sternfeld ([4], 1981), Haydon, Odell and Sternfeld ([5], 1981), among many other things, gave Maurey's theorem different proofs. Borwein and Sims ([6], 1984), and Domínguez Benavides ([7], 1996) proved the same conclusion holds for $c_{0}(\Gamma)$. Fuster and Sims $([8], 1998)$ showed that $c_{0}$ fails to have the fixed point property and conjectured that every closed bounded convex subset of $c_{0}$ with the fixed point property is weakly compact. An example of Pineda ([9], 2003) showed that the fixed point property in $c_{0}$ can not go beyond the class of all nonempty weakly compact convex subsets, even if to a compact convex subset in some topology slightly weaker than the weak topology. An affirmative answer to this conjecture was given by Dowling, Lennard and Turett ([10], 2004).

In this paper, we first show that for every mapping $f$ from a metric space $\Omega$ to itself which is continuous off a countable subset of $\Omega$, there exists a nonempty closed separable subspace $S \subset \Omega$ so that $\left.f\right|_{S}$ is again a self mapping on $S$. Therefore, the fixed point property of a closed convex set in a Banach space is separably determined. We then prove that every separable subspace of $c_{0}(\Gamma)$ (for any set $\Gamma$ ) is again lying in $c_{0}$. Making use of these results, we finally present a simple proof of the famous result: Every non-expansive selfmapping defined on a nonempty weakly compact convex set of $c_{0}(\Gamma)$ has a fixed point.

\section{Separable determination of the fixed point property}

In this section, we shall show that the fixed point properties for continuous (in particular, non-expansive) mappings is separably determined.

For subset $A$ in a topological space, we denote by $\bar{A}$, the closure of $A$.

Lemma 2.1. Suppose that $D$ is a nonempty closed subset of a metric space $\Omega$, and $f: D \rightarrow D$ is 
a continuous mapping apart from a countable set $D_{0} \subset D$. Then there exists a closed separable subset $S$ of $D$ such that $S$ is $f$-invariant, i.e, $f(S) \subset S$.

Proof. Given a nonempty separable subset $D_{1} \supset D_{0}$ of $D$, we first show

$$
f\left(D_{1}\right) \equiv\left\{f(x): x \in D_{1}\right\}
$$

is also separable. Note that $f$ is continuous at each point of $S_{1} \equiv D_{1} \backslash D_{0}$. Since $S_{1}$ is separable, there is a sequence $\left(x_{n}\right) \subset S_{1}$ which is dense in $S_{1}$. Given $y \in f\left(S_{1}\right)$, let $y=f(x)$ for some $x \in D_{1}$. Then density of $\left(x_{n}\right)$ in $S_{1}$ entails that there is a subsequence $\left(x_{n_{k}}\right)$ of $\left(x_{n}\right)$ so that $x_{n_{k}} \rightarrow x$ as $k \rightarrow \infty$. Continuity of $f$ at $x$ implies that $f\left(x_{n_{k}}\right) \rightarrow f(x)$. Thus,

$$
y=f(x) \in \overline{\left(f\left(x_{n_{k}}\right)\right)} .
$$

Since $y$ is arbitrary, we obtain that

$$
f\left(S_{1}\right) \subset \overline{\left(f\left(x_{n}\right)\right)}
$$

Therefore, $f\left(S_{1}\right)$ is separable. Consequently,

$$
f\left(D_{1}\right)=f\left(S_{1}\right) \cup f\left(D_{0}\right)
$$

is separable.

Since

$$
D_{2} \equiv \overline{D_{1} \cup f\left(D_{1}\right)}
$$

is separable, by iterating the procedure above, we know $f\left(D_{2}\right)$ is separable. Assume that $n$ separable subsets

$$
D_{1}, D_{2}, \cdots, D_{n} \text { with } D_{j}=\overline{D_{j-1} \cup f\left(D_{j-1}\right)}
$$

for $j=2, \cdots, n$ have been constructed. Next, let

$$
D_{n+1}=\overline{D_{n} \cup f\left(D_{n}\right)} .
$$

Then $D_{n+1}$ is again separable. Now, we obtain an increasing sequence $\left(D_{n}\right)$ of separable closed subsets od $D$ with

$$
D_{n+1}=\overline{D_{n} \cup f\left(D_{n}\right)} \quad \text { for all } n \in \mathbb{N} .
$$

Finally, let

$$
D_{\infty}=\overline{\bigcup_{n=1}^{\infty} D_{n} .}
$$

Then $D_{\infty}$ is again separable. We claim $f\left(D_{\infty}\right) \subset D_{\infty}$. Since

$$
D_{0} \cup f\left(D_{0}\right) \subset D_{n} \quad \text { for all } n \geq 1,
$$


We get $\overline{f\left(D_{0}\right)} \subset D_{\infty}$. Since

$$
f\left(D_{\infty}\right)=f\left(\overline{\bigcup_{n=1}^{\infty} D_{n} \backslash D_{0}}\right) \cup f\left(\overline{D_{0}}\right),
$$

and since $\bigcup_{n=1}^{\infty} D_{n} \backslash D_{0}$ is dense in $D_{\infty} \backslash D_{0}$, continuity of $f$ on $\bigcup_{n=1}^{\infty} D_{n} \backslash D_{0}$ allows us to show only that $f\left(\bigcup_{n=1}^{\infty} D_{n}\right) \subset D_{\infty}$. For any fixed $x \in \bigcup_{n=1}^{\infty} D_{n}$, there is $m \in \mathbb{N}$ so that $x \in D_{m}$. Thus, by definition of $D_{n}$ we obtain

$$
f(x) \in f\left(D_{m}\right) \subset D_{m+1} .
$$

Consequently,

$$
f\left(\bigcup_{n=1}^{\infty} D_{n}\right) \subset \bigcup_{n=1}^{\infty} D_{n} \subset D_{\infty} .
$$

We finish the proof by taking $S=D_{\infty}$.

The next lemma is a convex set version of Banach spaces of Lemma 2.1.

Lemma 2.2. Suppose that $D$ is a nonempty closed convex subset of a Banach space $X$, and $f$ : $D \rightarrow D$ is a continuous mapping apart from a countable set $D_{0} \subset D$. Then there exists a closed separable convex subset $C$ of $D$ such that $C$ is $f$-invariant, i.e, $f(C) \subset C$.

Proof. Choose any closed convex separable subset $D_{1}$ of $D$ with $D_{1} \supset D_{0}$. Then we see

$$
f\left(D_{1}\right) \equiv\left\{f(x): x \in D_{1}\right\}
$$

is also separable. Hence,

$$
D_{2} \equiv \overline{\mathrm{Co}}\left[D_{1} \cup f\left(D_{1}\right)\right]
$$

is closed convex and separable, where $\overline{\mathrm{co}}(A)$ stands for the closed convex hull of $A$, i.e. the closure of the set

$$
\operatorname{co}(A) \equiv\left\{\sum_{j=1}^{n} \lambda_{j} a_{j}: n \in \mathbb{N}, a_{j} \in A, \lambda_{j} \geq 0 \text { with } \sum_{j=1}^{n} \lambda_{j}=1\right\} .
$$

Generally, let

$$
D_{n+1}=\overline{\mathrm{co}}\left[D_{n} \cup f\left(D_{n}\right)\right], n \in \mathbb{N} .
$$

By an argument as the same as the one of the proof of Lemma 2.1, we observe that $\left(D_{n}\right)$ is an increasing sequence of closed convex separable subsets of $D$. Let

$$
D_{\infty}=\overline{\mathrm{co}}\left[\bigcup_{n=1}^{\infty} D_{n}\right] .
$$


Then $D_{\infty}$ is again closed convex and separable. Since $\left(D_{n}\right)$ is an increasing sequence of closed convex separable subsets, we see $D_{\infty}=\overline{\bigcup_{n=1}^{\infty} D_{n}}$. Note

$$
D_{0} \cup f\left(D_{0}\right) \subset D_{n} \quad \text { for all } n \geq 1 \text {. }
$$

Since

$$
f\left(D_{\infty}\right)=f\left(\overline{\bigcup_{n=1}^{\infty} D_{n} \backslash D_{0}}\right) \cup f\left(\overline{D_{0}}\right),
$$

and since $\bigcup_{n=1}^{\infty} D_{n} \backslash D_{0}$ is dense in $D_{\infty} \backslash D_{0}$, continuity of $f$ on $\bigcup_{n=1}^{\infty} D_{n} \backslash D_{0}$ allows us to show only that $f\left(\bigcup_{n=1}^{\infty} D_{n}\right) \subset D_{\infty}$. For any fixed $x \in \bigcup_{n=1}^{\infty} D_{n}$, there is $m \in \mathbb{N}$ so that $x \in D_{m}$. Thus, by definition of $D_{n}$, we obtain

$$
f(x) \in f\left(D_{m}\right) \subset D_{m+1} .
$$

Consequently,

$$
f\left(\bigcup_{n=1}^{\infty} D_{n}\right) \subset \bigcup_{n=1}^{\infty} D_{n} \subset D_{\infty} .
$$

Therefore, $C \equiv D_{\infty}$ is $f$-invariant.

Recall that a nonempty closed convex set $D$ of a Banach space $X$ is said to have the (respectively, weak) fixed point property, if for every nonempty closed bounded (respectively, weakly compact) convex subset $C$ of $D$, and for every non-expansive mapping $f$ defined on $C$, there is a point $x_{0} \in C$ so that $f\left(x_{0}\right)=x_{0}$. The following theorem states that the fixed point property and the weak fixed point property are separably determined.

Theorem 2.1. Both the fixed point property and the weak fixed point property of a closed convex set $D$ of a Banach space X (in particular, the whole Banach space X) are separably determined, i.e. the (respectively, weak) fixed point property is equivalent to that for each nonempty closed bounded (respectively, weakly compact) convex separable subset $C$ of $D$, and for every non-expansive mapping $f$ defined on $C$, there is a point $x_{0} \in C$ so that $f\left(x_{0}\right)=x_{0}$.

Proof. Suppose that $E$ is a nonempty closed convex subset of a Banach space $X$, that $D \subset E$ is a nonempty closed bounded convex set (respectively, nonempty weakly compact convex set), and that $f$ is a non-expansive mapping defined on $D$. Since $f$ is 1-Lipschitz, it necessarily fulfills that assumption of $f$ defined as in Lemma 2.2. Therefore, there is a nonempty closed separable convex (respectively, weakly compact) subset $C$ of $D$, so that $\left.f\right|_{C}$ (the restriction of $f$ from $D$ to $C$ ) is also a self-mapping. If $\left.f\right|_{C}$ has a fixed point, then $f$ has a fixed point. Thus, we have shown that if every non-expansive mapping defined on a nonempty closed bounded (respectively, weakly compact) convex separable subset has a fixed point, then every such mapping defined on a nonempty closed bounded (respectively, weakly compact) convex subset has a fixed point, which complete our proof. 
Remark 2.1. Given a Banach space $X$, we use $\mathcal{C}$ to denote a specific class of nonempty closed bounded convex sets of $X$, and $M(\mathcal{C})$ a specific class of self-mappings defined on some element $\mathcal{C}$ of $\mathcal{C}$. Following are fundamental examples of $\mathcal{C}$ and $M(\mathcal{C})$.

$$
\begin{gathered}
\mathcal{C}=\{C \subset X \text { nonempty, closed bounded and convex }\}, \text { and } \\
M(\mathcal{C})=\{f: C \rightarrow C \text { nonexpansive for some } C \in \mathcal{C}\} ; \\
\mathcal{W}=\{C \subset X \text { nonempty, weakly compact and convex }\} \text { and } \\
M(\mathcal{W})=\{f: C \rightarrow C \text { nonexpansive for some } C \in \mathcal{W}\} ; \\
\mathcal{C}=\{C \subset X \text { nonempty, closed bounded and convex }\}, \text { and } \\
T(\mathcal{C})=\{f: C \rightarrow C \text { continuous affine for some } C \in \mathcal{C}\} .
\end{gathered}
$$

We say that $X$ has the fixed point property for $M(\mathcal{C})$ provided every $f \in M(\mathcal{C})$ has a fixed point. There are many possibilities. For example, if $\mathcal{C}$ consists of all nonempty closed bounded convex set of $X$, and if $M(\mathcal{C})$ denotes class of non-expansive self-mappings, each of which defined on an element $C$ of $\mathcal{C}$, then $X$ has the fixed point property for $M(\mathcal{C})$ is equivalent to that $X$ has the fixed point property; if $\mathcal{W}$ consists of all nonempty weakly compact convex set of $X$, and if $M(\mathcal{W})$ denotes class of non-expansive self-mappings, each of which defined on an element $C$ of $\mathcal{W}$, then $X$ has the fixed point property for $M(\mathcal{W})$ is equivalent to that $X$ has the weak fixed point property. Keeping this in mind, we can observe that the following more general result is true.

Theorem 2.2. Suppose that $X$ is a Banach space. Then the fixed point property for $M(\mathcal{C})$ is separably determined if every element in $M(\mathcal{C})$ is continuous off at most a countable subset of its domain.

The following notions can be found in [11] and [12]. A closed convex set $D$ of a Banach space $X$ is said to have the super fixed point property provided that every nonempty closed bounded convex set $C$ of another Banach space $Y$ has the fixed point property whenever $C$ is finitely representable in $D$, i.e. for all $\varepsilon>0$ and for any affinely indenpendent finite set $\left\{x_{0}, x_{1}, \cdots, x_{n}\right\} \subset C$ (which is equivalent to $x_{1}-x_{0}, x_{1}-x_{0}, \cdots, x_{n}-x_{0}$ are linearly independent) there exist an affinely independent finite set $\left\{y_{0}, y_{1}, \cdots, y_{n}\right\} \subset D$ and an affine mapping $T: \operatorname{span}\left\{x_{0}, x_{1}, \cdots, x_{n}\right\} \rightarrow \operatorname{span}\left\{y_{0}, y_{1}, \cdots, y_{n}\right\}$ so that

$$
\begin{gathered}
T \operatorname{co}\left\{x_{0}, x_{1}, \cdots, x_{n}\right\}=\operatorname{co}\left\{x_{0}, x_{1}, \cdots, x_{n}\right\}, \text { and } \\
(1-\varepsilon)\|x-y\| \leq\|T x-T y\| \leq(1+\varepsilon)\|x-y\|, x, y \in \operatorname{co}\left\{x_{0}, x_{1}, \cdots, x_{n}\right\} .
\end{gathered}
$$

Analogously, we have the following theorem.

Theorem 2.3. A nonempty closed convex set of a Banach space X has the super fixed point property if and only if every nonempty closed convex separable subset of it has the super fixed point property. 


\section{A simple proof of $c_{0}(\Gamma)$ admitting the weak fixed point prop- erty}

As an application of Theorem 2.4, and making use of Maurey's classical theorem [3], we give a simple proof $c_{0}(\Gamma)$ has the weak fixed point property for any set $\Gamma$. Before doing this, we need some preparation.

Recall a mapping $U$ from a Banach space $X$ to another Banach space $Y$ is said to be a linear isometry provided

$$
\|U x-U y\|=\|x-y\|, \quad \text { for all } x, y \in X .
$$

In this case, we also say that $X$ is linear isometric to a subspace of $Y$. If the linear isometry is surjective, then we call the two spaces "isometric". Since $U$ is linear, the equation above is equivalent to

$$
\|U x\|=\|x\|, \quad \text { for all } x \in X .
$$

The following result states that every separable subset of the space $c_{0}(\Gamma)$ is again in $c_{0}$.

Lemma 3.1. Every separable subspace of $c_{0}(\Gamma)$ is linearly isometric to a subspace of $c_{0}$.

Proof. Suppose that $E$ is a separable subspace of $c_{0}(\Gamma)$. We choose any sequence $\left\{x_{n}\right\}_{n \in \mathbb{N}} \subset$ $E$, which is a dense sequence in $E$; and put

$$
\Gamma_{n}=\operatorname{supp} x_{n} \equiv\left\{\gamma \in \Gamma: x_{n}(\gamma) \neq 0\right\} \text { and } \Gamma_{\infty}=\cup_{n=1}^{\infty} \Gamma_{n} .
$$

Then by definition of $c_{0}(\Gamma), \Gamma_{n}$ is countable for every $n \in \mathbb{N}$. Therefore, $\Gamma_{\infty}$ is again a countable subset of $\Gamma$. Let $\chi_{A}$ be the mapping sending every set $A \subset \Gamma$ to its characteristic function, i.e. $\chi_{A}(x)=1$, if $x \in A$; $=0$, otherwise, and let $P_{A}: c_{0}(\Gamma) \rightarrow c_{0}(\Gamma)$ be the natural projection defined by

$$
P_{A}(x)=x \circ \chi_{A}=\left(x(\gamma) \cdot \chi_{A}(\gamma)\right)_{\gamma \in \Gamma}
$$

Then

$$
P_{\Gamma_{\infty}}\left(c_{0}(\Gamma)\right)=c_{0}(\Gamma) \circ \chi_{\Gamma_{\infty}}=\left\{x \circ \chi_{\Gamma_{\infty}}: x \in c_{0}(\Gamma)\right\}
$$

is linearly isometric to $c_{0}\left(\Gamma_{\infty}\right)$. Since $\Gamma_{\infty}$ is countable, it is linearly isometric to $c_{0}$, if the cardinality $\alpha=\infty$ of $\Gamma_{\infty}$; and to $\ell_{\infty}^{\alpha}$ if $\infty>\alpha$. Therefore, $c_{0}\left(\Gamma_{\infty}\right)$ is linearly isometric to a subspace of $c_{0}$. Therefore, up to a linear isometry, we have

$$
c_{0} \supset c_{0}\left(\Gamma_{\infty}\right) \cong c_{0}(\Gamma) \cdot \chi_{\Gamma_{\infty}} \supset\left\{x_{n}\right\},
$$

where $c_{0}\left(\Gamma_{\infty}\right) \cong c_{0}(\Gamma) \circ \chi_{\Gamma_{\infty}}$ means that $c_{0}(\Gamma) \circ \chi_{\Gamma_{\infty}}$ is linearly isometric to $c_{0}\left(\Gamma_{\infty}\right)$. This and density of $\left\{x_{n}\right\}$ in $E$ deduce

$$
c_{0} \supset c_{0}\left(\Gamma_{\infty}\right) \cong c_{0}(\Gamma) \cdot \chi_{\Gamma_{\infty}} \supset \overline{\left(x_{n}\right)}=E,
$$

i.e. $E$ is linearly isometric to a subspace of $c_{0}$. 
We shall use Maurey's theorem [3] that $c_{0}$ has the weak fixed point property to prove the following theorem.

Theorem 3.1. For any nonempty set $\Gamma, c_{0}(\Gamma)$ has the weak fixed point property.

Proof. By Maurey's theorem, without loss of generality, we can assume that $\Gamma$ is uncountable. Suppose that $D$ is a nonempty weakly compact convex subset of $c_{0}(\Gamma)$, and $f: D \rightarrow D$ is a non-expansive mapping. According to Lemma 2.2, there is a nonempty closed convex separable subset $C$ of $D$ so that $\left.f\right|_{C}$ is again a self-mapping on $C$. Since $D$ is a weakly compact convex subset, $C$ is weakly compact. Since $C$ is separable, $E \equiv \overline{\operatorname{span}}(C)$ is a separable subspace of $c_{0}(\Gamma)$. According to Lemma 3.1, there is a linear isometry $T$ from $E$ into $c_{0}$. Since $T$ is linear and continuous, it is necessarily weakly continuous. Consequently, $T C$ is again convex, separable and weakly compact in $c_{0}$. Let

$$
F=T \circ f \circ T^{-1}: T C \rightarrow T C .
$$

Then it satisfies that for all $x, y \in T C$

$$
\begin{aligned}
\|F(x)-F(y)\| & =\left\|T \circ f \circ T^{-1}(x)-T \circ f \circ T^{-1}(y)\right\| \\
& =\left\|f \circ T^{-1}(x)-f \circ T^{-1}(y)\right\| \\
& \leq\left\|T^{-1}(x)-T^{-1}(y)\right\| \\
& =\|x-y\| .
\end{aligned}
$$

Thus, $F$ is also a nonexpansive mapping from the nonempty weakly compact convex set $T C$ to itself. By Maurey's fixed point theorem there exists $y_{0} \in T C$ so that $F\left(y_{0}\right)=y_{0}$; or, equivalently, $f\left(x_{0}\right)=x_{0}$, where $x_{0}=T^{-1} y_{0} \in C$. We have shown that there is a fixed point $x_{0} \in C \subset D$ for the mapping $\left.f\right|_{C}$, hence, $f$.

\section{Acknowledgments}

The authors would like to thank people in the functional analysis seminar of Xiamen University for their helpful conversations.

\section{References}

[1] W. A. Kirk, B. Sims (Eds.). Handbook of metric fixed point theory. Kluwer Academic Publ., Dordrecht, 2001.

[2] J. Lindenstrauss. On nonseparable reflexive Banach space. Bull. Amer. Math. Soc., 1966, 72 : 967-970.

[3] B. Maurey. Points fixes des contractions de certains faiblement compacts de L1. Seminaire d'Analyse Fonctionelle, 1980-1981. Centre de Mathematiques, Ecole Polytech., Palaiseau, 1981, Exp. No. VIII, 19 pp. MR 83h:47041.

[4] E. Odell, Y. Sternfeld. A fixed point theorem in $c_{0}$. Pacific J. Math., 1981, 95(1): 161-177. 
[5] R. Haydon, E. Odell, Y. Sternfeld. A fixed point theorem for a class of star-shaped sets in $c_{0}$. Israel J. Math., 1981, 38: 75-81.

[6] Borwein, Jonathan M., B. Sims. Non-expansive mappings on banach lattices and related topics. Houston J. Math., 1984, 10: 339-356.

[7] T. Domínguez Benavides. A geometrical coefficient implying the fixed point property and stability results. Houston J. Math., 1996, 22(4): 835-849.

[8] E.L. Fuster, B. Sims. The fixed point property in $c_{0}$. Canad. Math. Bull., 1998, 41(4): 413-422.

[9] M.J. Pineda. The fixed point property in Banach spaces containing a copy of $c_{0}$. Abstr. Appl. Anal., 2003, 35: 183-192.

[10] P.N. Dowling, G.J. Lennard, B. Turett. Weak compactness is equavilent to the fixed point property in $c_{0}$. Proc. Amer. Math. Soc., 2004, 132(6): 1659-1666.

[11] L. Cheng, Q. Cheng, B. Wang, W. Zhang. On super-weakly compact sets and uniformly convexifiable sets. Studia Math., 2010, 199(2): 145-169.

[12] L. Cheng, Q. Cheng, J. Zhang. On super fixed point property and super weak compactness of convex subsets in Banach spaces. J. Math. Anal. Appl., 2015, 428(2): 1209-1224. 